Research Article

\title{
Innovación en la docencia del periodismo a través de la ciencia de datos
}

\section{Innovation in journalism education through data science}

\author{
Adolfo Antón Bravo ${ }^{*}$ y Ana Serrano Tellería ${ }^{2}$ \\ ${ }^{1}$ Centro de Ciencias Humanas y Sociales-CCHS - Consejo Superior de Investigaciones Científicas-CSIC, \\ C/. Albasanz, 26-28, 28037 Madrid. \\ ${ }^{2}$ Universidad de Castilla-La Mancha, Facultad de Comunicación. Plaza de la libertad de expresión, \\ Aulario Polivalente, s/n, Campus Cuenca, 16071 Cuenca. \\ *Correspondencia: adolflow@gmail.com
}

Resumen: El periodismo de datos moderno nace en 2008 con la confluencia de tres factores fundamentales: los renovados formatos de la Web, con HTML5 y CSS3 a la cabeza, la abundancia de software libre disponible para cada una de las etapas del proceso de investigación con datos y la apertura de portales de datos abiertos de los gobiernos. Para los periodistas uno de los retos fundamentales de este cambio tecnológico ha sido encontrar la formación adecuada. Existen diversas experiencias y enfoques en la educación periodística con datos; en este artículo se examina la docencia del periodismo de datos en España a través del análisis de las propuestas docentes en siete másteres impartidos por universidades españolas.

Palabras clave: periodismo de datos; visualización de datos; ciencia de datos; periodismo; educación.

\begin{abstract}
Data journalism was born in 2008 with the confluence of three fundamental factors: the renewed formats of the Web, with HTML5 and CSS3 at the forefront, the abundance of free software available for every stage of the data research process, and the opening of government open data websites. For journalists, one of the fundamental challenges of this technological change has been to find the right training. There are diverse experiences and approaches to journalism education with data; this article examines the teaching of data journalism in Spain through the analysis of teaching proposals in seven master's degrees offered by Spanish universities.
\end{abstract}

Keywords: data journalism; data visualisation; data science; journalism; education.

\section{Introducción}

El periodismo siempre ha trabajado con datos. Sin embargo, en los últimos años, han cobrado importancia el periodismo de datos y la visualización de datos, prácticas y metodologías que pretenden hacer inteligibles las grandes cantidades de datos disponibles en la actualidad.

Abordamos en este artículo la innovación docente en esta área y, en concreto, la inclusión de estas asignaturas o asignaturas directamente relacionadas con el periodismo y la visualización de datos en las universidades españolas. Para ello recogemos la información disponible en sus páginas web de los programas de grado o posgrado que abordan estas temáticas. Este artículo pretende mostrar el recorrido seguido por el estado de la cuestión en el aprendizaje formal y 
reglado de periodismo de datos en las universidades de España y los debates que se producen a escala internacional.

\section{Marco teórico}

El periodismo de datos moderno nace en 2008 con la confluencia de tres factores fundamentales: los renovados formatos de la Web con HTML5, CSS3 a la cabeza, la abundancia de software libre disponible para cada una de las etapas del proceso de investigación de los datos y la apertura de portales de datos abiertos de los gobiernos. Es en 2008 cuando se funda Five Thirty Eight y en 2009 el Datablog del The Guardian (Bradshaw, 2017).

Según Rogers (2012) y Gray et al. (2012) el periodismo de datos o periodismo guiado por datos (Data Driven Journalism, DDJ) ha sido definido como el periodismo que se basa en grandes conjuntos de datos. Paul Bradshaw (2010) señala el increíble acontecimiento de 2010 con Wikileaks, una filtración de información de cables diplomáticos de EE.UU. entre los que se incluían datos sobre las guerras de Afganistán o Iraq. Fue la primera y más sonada de las filtraciones de una serie protagonizada por el Consorcio Internacional de Periodistas de Investigación (International Consortium of Investigative Journalists, ICIJ) Luxembourg Leaks y Swiss Leaks, en 2014 y 2015 respectivamente, sobre evasiones fiscales, con dos veces más datos que Wikileaks; Offshore Leaks en 2013, 150 veces más grande; y Panama Papers (ICIJ, 2017) en 2016, más grande que todos los anteriores juntos. Un fenómeno que incluso tuvo su eco en España (Cordobés, 2017).

Desde el principio, el periodismo de datos presenta dos características fundamentes. Por un lado, se trata de una disciplina que se ha ejercido y aprendido sobre la práctica, tomando como referente tanto el periodismo de precisión (Precision Journalism) de Philip Meyer (1991) como las técnicas de periodismo asistido por ordenador (Computer Assisted Reporting) (Meyer, 2002) o cualquier otro documento científico o manual que pudiera aprovecharse y con lentitud se va trasladando a los programas universitarios. Por otro, una convivencia con la visualización de datos entendida desde una doble perspectiva que permite la exploración de grandes volúmenes de datos, pero también que participa de los formatos de salida de los proyectos de periodismo de datos.

\subsection{El reto de enseñar periodismo de datos en la universidad}

Davies y Cullen (2016) señalaban que se había ido muy lento en la inclusión de estas prácticas en el currículo de la educación en periodismo:

“Ha sido lenta la inclusión de las ideas clave de los pioneros en el plan de estudios de la enseñanza diaria del periodismo. "Mi mensaje para otros interesados en el progreso del periodismo de datos en la academia es que estamos trabajando en ello".

Para revertirlo se propuso realizar una investigación académica que revisara otras publicaciones que hubieran abordado ese tema y una serie de entrevistas a (treinta y cinco) personas del ámbito académico de Australia que le contaran qué estaban haciendo al respecto. La principal conclusión que extrae es que no es la única persona que está preguntándose si la docencia del periodismo de datos se está realizando correctamente y considera que por lo pronto se deberían incluir herramientas y técnicas en el currículo universitario para ayudar a llevar al periodismo de datos en los planes de estudio de periodismo.

El caso de Davies y Cullen (2016) es el de muchos profesores (o colaboradores docentes u otras figuras) que contratan las universidades para llevar esas prácticas novedosas a la formación universitaria y se muestran preocupados por los resultados. Además, en aquella época, Davies consideraba que las características especiales que presenta el periodismo de datos no están cubiertas por periodistas que hayan vivido tanta experiencia como la que la enseñanza de estas materias en su amplio espectro requiere (Hanusch et al., 2016). 
En su investigación los participantes coincidían en la necesidad de aumentar las habilidades de los periodistas, pero reconocían que, ante la falta de fondos para que profesionales de amplia experiencia les enseñaran, abogaban por el aprendizaje autónomo, lo cual se enfrenta con la falta de tiempo, la gran cantidad de software para aprender y los distintos enfoques posibles para dominar las diferentes materias. Por el lado de los estudiantes también observa que llegan al mundo del periodismo y la visualización de datos con algunos conocimientos relacionados, pero son tan variados y dispares que resulta difícil encontrar el itinerario correcto. Además, se constata un problema social que denomina de "aversión a las matemáticas", algo que en su opinión debe atajarse de raíz.

De hecho, en su curso de periodismo de datos realizado en $2016^{1}$ propone una dinámica grupal en la primera clase donde confiesan su aversión en grupo y acuerdan convivir con el miedo para superarlo. Según los cuestionarios realizados al final del curso, la experiencia había sido satisfactoria porque, aunque fueran conscientes de haber resuelto mínimamente las complejidades del periodismo de datos de vanguardia, al abordar los temores y trabajarlos desde la raíz se reconocen preparados para una vida de aprendizaje. Uno de los participantes del estudio proponía como forma de superación algo tan simple y tan necesario como una buena guía de los pasos que hay que dar, de los inconvenientes que se pueden encontrar y de los trucos con los que los superaron.

\subsection{Distintas estrategias}

Esta preocupación ha seguido cobrando el interés por parte de las personas relacionadas con el periodismo y la visualización de datos. El sitio web datajournalism.com, realizado por el European Journalism Center, publicó en agosto de 2019 un artículo titulado "7 countries, 9 teachers: a dossier of data journalism teaching strategies. What are the most effective ways to introduce students to data?" (7 países, 9 profesores: un dossier de las estrategias docentes de periodismo de datos) en el que lamentablemente no se mencionaba el caso de España, pero sí que se apuntaban cuestiones comunes (Heravi, 2018).

El artículo comienza con lo que Davies había señalado como la principal lacra de las personas que trabajan en periodismo de datos, la aversión a las matemáticas, a lo que cabría preguntarse si no habría que añadir "aversión a usar un ordenador" como lo usan los científicos de datos, por ejemplo, lo cual implica como solución, en ambos casos, abrazar un software que suplante la pericia matemática, computacional, algorítmica o de otro tipo para hacer "fácil" el trabajo del periodista de datos, ya sea el de recolectar, extraer, limpiar, investigar o crear gráficos o mapas. Tal como dijo Philip Meyer $(1970,2002)$ al definir el periodismo de precisión y así se ha ido narrando en la producción científica y literaria del mundo del periodismo de precisión, del CAR (Computer Assisted Reporting, periodismo asistido por ordenador) o del DDJ (Data Driven Journalism, periodismo guiado por datos), para saber encontrar e informar con precisión en las historias (McGregor, 2012) de la actual sociedad de los datos (sociedad de la información), necesitas esos conocimientos.

Paul Bradshaw (2018) señala igualmente en "Teaching Datajournalism slow" que el principal problema con el que cuentan los alumnos de periodismo o los profesionales es la falta de conocimientos matemáticos. En sus formaciones iniciales, en 2010, abordaba un aprendizaje que empezaba por la hoja de cálculo, datos y funciones. Pero en 2016 decide cambiar y comienza con unos datos ya trabajados con los que realizar visualizaciones que "cuenten una historia". Esto le funcionó mejor porque había motivación y resultados inmediatos en "apenas una hora" y consiguió que los estudiantes estuvieran más receptivos para empezar a trabajar con los datos. A la siguiente sesión volvió a la hoja de cálculo y, contrariamente a lo que ocurría en otras ocasiones, ya no se percibieron "operaciones matemáticas" sino "cómo encontrar historias que contar".

${ }^{1}$ Business and Data Journalism, JOU3209, Edith Cowan University, Australia. https://www.ecu.edu.au/handbook/unit?id=JOU3209\&year=2018 
Además, produjo el libro Data Journalism Heist (Bradshaw, 2013) como material docente de apoyo.

\subsection{La pirámide invertida del periodismo de datos}

El recorrido inicial de la formación y aprendizaje de periodismo de datos fue compilado por de Paul Bradshaw con cinco pasos en forma de pirámide invertida (Bradshaw, 2011):

1. Compile, compilar.

2. Clean, limpiar.

3. Context, contextualizar.

4. Combine, combinar.

5. Communicate, comunicar.

Figure 1. Pirámide invertida del Periodismo de Datos.

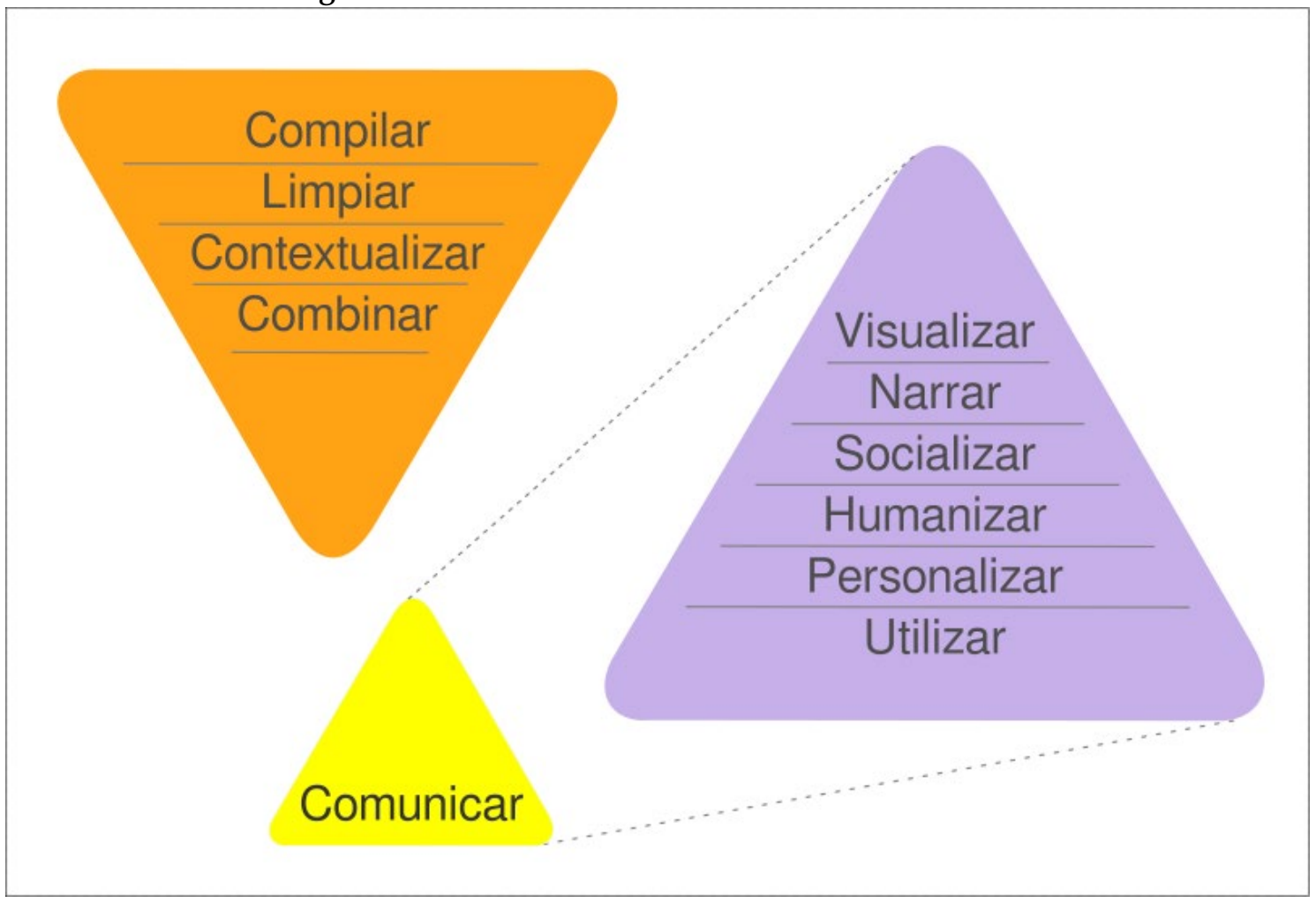

Fuente: Adaptación propia CC-BY-SA basada en original de Paul Bradshaw (2011).

Davies, señala en su estrategia de trabajo (Davies \& Cullen, 2016) el hecho de introducir una actividad de interrogatorio de los datos en el primer año les refuerza sus habilidades troncales, lo mismo que entrevistar o contrastar hechos. Mientras que para Nouha Belaid, de la Central University of Tunisia, el principal reto que observa para el lento desarrollo del lento desarrollo del periodismo de datos en su país lo explica con el enigma del huevo y la gallina: sin una educación en datos adecuada no habrá más personas que sepan trabajar con datos y sin personas que sepan trabajar con datos no habrá trabajos que enseñar ni profesionales que enseñen estas prácticas.

Un apunte muy novedoso lo realiza Dr. Reselyn Du de la Hong Kong Baptist University para quien el periodismo de datos es una forma de distinguir a los periodistas, profesionales de la comunicación, de la comunicación que se realiza en el mundo de las redes sociales. Dado que ahora cualquier ciudadano puede, con su teléfono móvil, participar de la difusión y retransmisión de noticias, audios o vídeos, el periodismo guiado por datos cuenta con un valor añadido, una cualidad profesional que se puede asociar al propio ejercicio del periodismo. Tanto es así que 
Irene Jay Liu ${ }^{2}$ comentaba en 2013 para el Pacific Media Center que el periodismo de datos podía justificar la continuación de la existencia de un periodismo profesional, amenazado por la convergencia mediática, cierres de redacciones o desaparición de medios regionales.

Roselyn realizó una encuesta a 121 estudiantes de su universidad (Yang \& Du, 2016) para evaluar las mejores estrategias para el estudio del periodismo de datos y concluyó que, aunque tienen muchas ganar por aprender de datos, no entienden cómo se recogen, analizan o interpretan, no conocen las herramientas para hacerlo y a la mitad de quienes lo conocen les resulta poco atractivo. Es decir, aunque reconocieran la importancia de los datos, la mayoría mostró mínimo interés por adquirir habilidades en el tratamiento de datos, lo cual Roselyn asocia al hecho de no haber desarrollado ese interés desde el inicio de los programas de periodismo. Por tanto, la principal medida que habría que tomar tendría que ser dotar a estos programas de estas materias y considerar la alfabetización en datos como una inversión más que como un coste.

Jeff Kelly Lowenstein de la Grand Valley State University de EE.UU. resume su experiencia docente en seis consejos (Treadwell et al., 2016). El primero es recomendar a los estudiantes que participen en los concursos que organiza la asociación de periodistas y editores de investigación de la asociación de periodistas y editores de investigación, Investigative Reporters and Editors (IRE) ya que muchos de los proyectos que se desarrollan aportan la metodología con la que se han realizado y, por tanto, se pueden replicar o hacer ingeniería inversa con su investigación y puede ser, además, una forma de entrar en contacto con todos esos profesionales y aprender de ellos. El segundo aboga por hacer cosas progresivamente más complejas ya que los pequeños proyectos no han de quedarse pequeños, sino que constituyen la base para mayores y más ambiciosos proyectos. El tercero sitúa el fallo en el centro del aprendizaje, fundamental para aprender y crecer. El cuarto recomienda a los estudiantes se sientan cómodos con los primeros pasos de los distintos saberes y habilidades que han de tener y que vean cómo están interconectados. En quinto lugar, resulta fundamental combinar la paciencia, no perder la perspectiva y pensar en distintas opciones antes de elegir una en concreta. Por último, en sexto lugar, los datos nunca son la historia, sino que lo importante es cómo esas historias afecten a individuos o comunidades.

En Asia central, Anastasia Valeeva, de la American University of Central Asia en Bishek, Kyrgyzstam, apunta un reto que no había previsto inicialmente al formar a profesionales de periodismo de datos y era el hecho de que hubiera otros profesionales de la redacción que entendieran los principios del periodismo de datos de cara a que fueran capaces de trabajar conjuntamente. Por ello resolvieron que había que mejorar la cooperación con otros departamentos universitarios de sociología, estadística o tecnologías de la información para extender el conocimiento sobre el periodismo de datos más allá del propio periodismo.

Por su parte, Bahareh Heravi, del University College Dublin (UCD) de Irlanda, comenta que pese al excepcional posicionamiento de su país en el sector de las tecnologías de la información y del conocimiento, como sede de muchas empresas tecnológicas de la región europea, no ha desarrollado tanto como Reino Unido o Alemania el periodismo de datos. Para elegir el tipo de formación a desarrollar realizó una encuesta durante cinco meses en colaboración con Mirko Lorenz de Datawrapper y otros medios en la que pregunta qué tipo de periodismo de datos se practica para ver qué tipo de estudio se necesita, la "Global Data Journalism Survey" (Heravi, 2018) donde reciben 181 respuestas de 43 países. Luego estudió alrededor de 220 cursos relacionados con periodismo de datos de todo el mundo para concluir que debía realizar un posgraduado en periodismo de datos que denominaron UCD Data Journalism ProfCert ${ }^{3}$ en 2017 en el que combinaron periodismo de datos con análisis de datos cuantitativos, estadística y $\mathrm{R}^{4}$,

\footnotetext{
${ }^{2}$ Editora de noticias en la sección de datos para Thomson Reuters de Hong Kong. https://www.irenejayliu.com/

${ }^{3}$ https://sisweb.ucd.ie/usis/!W_HU_MENU.P_PUBLISH?p_tag=PROG\&MAJR=W386

${ }^{4} \mathrm{R}$ : Entorno de software libre para el análisis estadístico y la visualización de datos.

https://www.r-project.org/
} 
para finalizar con un sitio web del proyecto desarrollado durante el curso $\mathrm{y}^{5}$ que, en la medida de lo posible, fueran publicados por algunos medios, como el "Personal Injury Claims in Ireland"6 o "Most dangerous cities for Gardai"7.

Figure 2. Resultados de la encuesta Global Data Journalism Survey.

Porcentaje de conocimientos en periodismo, análisis de datos, programación, estadística, ciencia de datos, aprendizaje automático y visualización de datos.

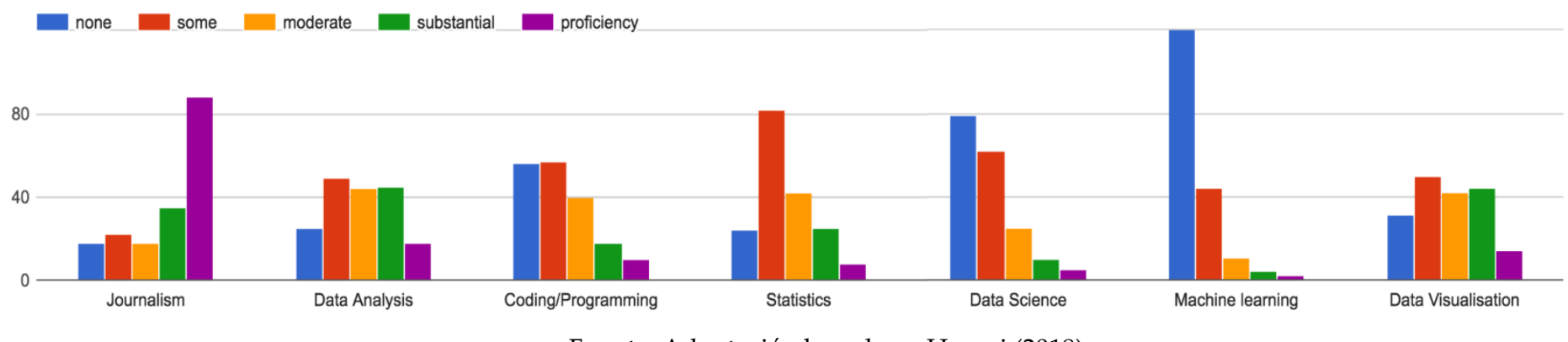

Fuente: Adaptación basada en Heravi (2018).

\subsection{La aversión a las matemáticas}

Linda Galligan (2013) resume que las matemáticas están menospreciadas en la educación actual lo que supone un problema doble de falta de competencias y de consciencia de ello. Pilar J. López-López (2019) recuerda cuando Bill Kovach y Tom Rosenstiel (2014) afirman que, "en el periodismo de investigación moderno el poder de análisis que permite la informática sustituye a menudo a la observación personal del reportero". López-López (2019) hace un repaso de la oferta de docencia en España relacionada con el periodismo de datos y tan solo encuentra en el "Máster de Periodismo de Investigación, Datos y Visualización" de la UNIR dos asignaturas relacionadas con esta línea, "La búsqueda de datos en el periodismo de investigación: minería de datos", y una optativa, "El periodista como programador". Quizás por ese reflejo en la falta de asignaturas que apuesten por aspectos técnicos propone en el capítulo 7 (López-López, 2019) una guía docente para el periodismo de datos con cinco bloques de los cuales uno se dedica a "Los algoritmos. Los lenguajes de programación". Seguía la línea que marcaba años antes la directora del máster donde observaba que "la oferta educativa española resulta insuficiente en este terreno" (Domínguez, 2014).

Charles Berret y Cheryl Phillips (2016) observaron que el común denominador de los perfiles que se buscaban en periodismo era el de tener experiencia en el trabajo con datos aunque a mayoría de los programas docentes no lo abordaban. Los autores concluían que los estudios de periodismo debían ampliar su temario para abordar las prácticas con datos y ordenadores como habilidades básicas, incorporar el periodismo de datos a estudios en curso y realizar nuevos programas específicos de periodismo guiado por datos.

Otros autores como Anderson (2013) han denominado "periodismo informático" (computational journalism) a los cambios que se están produciendo en las redacciones de los medios para trabajar con algoritmos, ciencias sociales y matemáticas, lo que en EE.UU. conocen como computational journalism o "periodismo informático", que bebe del periodismo de precisión de Meyer (1991) y se desarrolla durante el periodismo asistido por ordenador (Cox, 2000).

\footnotetext{
5https://newslab.ie/; https://newslab.ie/ddjucd/

6https://www.rte.ie/news/investigations-unit/2018/0424/956812-personal-injury-data/

7https://newslab.ie/ddjucd/what-are-the-most-dangerous-counties-for-gardai/
} 


\subsection{Los datos de España}

En España comienza en 2015 el pionero "Máster Propio en Periodismo de Investigación, Datos y Visualización", promovido por Unidad Editorial (El Mundo, Expansión, etc.) y la Universidad Rey Juan Carlos (URJC), con el apoyo de Google y con un equipo de profesionales de reconocido prestigio formado por Antonio Rubio (investigación en El Mundo), Marcos García (periodista independiente) y Mar Cabra, beca Fulbright en EE.UU. y abanderada del periodismo de datos desde el grupo de trabajo de periodismo de datos (GTPD) del centro cultural MedialabPrado perteneciente al Ayuntamiento de Madrid.

Se producía con este máster una evolución en la formación de periodismo de datos que primero se había realizado de manera informal, no reglada, con actividades del GTPD ue comenzó su andadura en octubre de 2011 consistentes en charlas, talleres, hackatones, barcamps, etc., a un máster en un entorno universitario reglado con una apuesta de varios actores implicados: academia, empresa de tecnologías de la información y empresa informativa.

Luego vinieron dos experiencias muy distintas entre sí, por un lado el "Máster en Periodismo de Investigación, Datos y Visualización" de la Universidad Internacional de la Rioja (UNIR), que copiaba el nombre al primero, y por otro el "Máster de Periodismo de Datos" realizado por el Centro Universitario Villanueva (CUV), adscrito a la Universidad Complutense de Madrid (UCM), ambos desaparecidos. El primero estaba dirigido por Marian Chaparro y coordinado por Juantxo Cruz y destacaba sobre los otros por la apuesta por la validación de ANECA (Agencia Nacional Española de Certificación Académica) y el hecho de ser fundamentalmente en línea.

El máster de URJC ha presumido de haber logrado la empleabilidad de sus alumnos en El Confidencial, La Sexta, La Vanguardia, La Nación (Argentina), haber participado en las investigaciones internacionales Futbol Leaks y Panama Papers, haber obtenido los premios Pulitzer 2017, APM, IPYS, Society for News Design y haber sido finalista de los premios Latam Digital Media.

Otro modelo de formación, el de aprendizaje informal, comienza en 2011 con el GTPD y se mantiene después de la creación del primer máster. El grupo es un compendio de personas de campos muy diversos y se producen algunas conexiones con otros proyectos como la Escuela de Datos (School of Data) de la Open Knowledge Foundation y con otros territorios como Barcelona, Pamplona o Almería. Dos de sus actividades se convierten en referente nacional: los Talleres de Producción de Periodismo de Datos (TPD, 2011-2019) y las Jornadas de Periodismo de Datos y Datos Abiertos (JPD), creadas por Karma Peiró y Mar Cabra. Además, en 2016 empieza el proyecto DataLab (2016-2019) que reúne todas estas actividades y establece múltiples alianzas en el campo de la ciencia de datos o DataScience (Anton, 2020) como por ejemplo el proyecto JournocodersMAD, R-Ladies, Python Madrid, Haskell o Emacs, entre otros.

Las mismas dudas que expresaba Davies y Cullen (2016) sobre el "mínimo tecnológico requerido" fueron compartidas por esta experiencia formativa. Mientras en 2013 y 2014 se trabajaba con herramientas de software que suplían esas carencias sin abordar la problemática de raíz y generando otras dependencias, a partir de 2015 se apostó por las tecnologías abiertas.

\section{Recogida de datos}

Para este artículo se han seleccionado siete másters y un curso que están en activo en España que parten del periodismo de datos o del periodismo. Se han descartado algunos másters cuyas temáticas podrían estar relacionadas: verificación de datos, visualización de datos o la ciencia de datos porque o bien son más específicos, y por tanto más parciales, o bien no abordan ni mínimamente en su conjunto el periodismo de datos. De algunos másters se han cuantificado todas las asignaturas, por entenderlas parte del recorrido del periodismo de datos, pero de otros se han seleccionado solo las que se consideran en esta disciplina. Por ejemplo, el uso de redes sociales ha quedado fuera porque no se aborda desde el plano de análisis de redes, recopilación 
de datos, trabajo con APIs, redes de conocimiento, grafos de conocimiento, análisis de sentimiento o cualquier otra perspectiva técnica avanzada.

\subsection{Máster en Periodismo de Investigación, Datos y Visualización}

Máster propio en Periodismo de Investigación, Datos y Visualización de la Universidad Rey Juan Carlos (URJC).

Tabla 1. Máster en Periodismo de Investigación, Datos y Visualización.

\begin{tabular}{clcc}
\hline Número & \multicolumn{1}{c}{ Módulo } & Créditos & Tipo \\
\hline 1 & Periodismo de Investigación & 9 & Obligatoria \\
\hline 2 & Periodismo de datos & 9 & Obligatoria \\
\hline 3 & Derecho a saber & 9 & Obligatoria \\
\hline 4 & Visualización de datos & 9 & Obligatoria \\
\hline 5 & Publicación & 9 & Obligatoria \\
\hline 6 & Trabajo Fin de Máster & 9 & Obligatoria \\
\hline 7 & Prácticas remuneradas 6 meses & 6 & Obligatoria \\
\hline & Fuente: Elaboración propia. & &
\end{tabular}

\subsection{Máster en Periodismo Digital y de Datos}

Máster universitario en Periodismo Digital y de Datos de la Universidad de Nebrija.

Tabla 2. Máster en Periodismo Digital y de Datos.

\begin{tabular}{clcc}
\hline Número & \multicolumn{1}{c}{ Módulo } & Créditos & Tipo \\
\hline 4 & $\begin{array}{l}\text { Programación, aplicaciones, herramientas y } \\
\text { soportes }\end{array}$ & 6 & Obligatoria \\
\hline 9 & Fuentes y tratamiento de datos & 4 & Obligatoria \\
\hline 10 & $\begin{array}{l}\text { Herramientas digitales para la visualización } \\
\text { y presentación de datos }\end{array}$ & 4 & Obligatoria \\
\hline
\end{tabular}

Fuente: Elaboración propia.

\subsection{Máster en Periodismo y Comunicación Digital: Datos y Nuevas narrativas}

Máster en Periodismo y Comunicación Digital: Datos y Nuevas Narrativas, de la Universitat Oberta de Catalunya (UOC).

Tabla 3. Máster en Periodismo y Comunicación Digital: Datos y Nuevas Narrativas.

\begin{tabular}{clcc}
\hline Número & \multicolumn{1}{c}{ Módulo } & Créditos & Tipo \\
\hline 1 & Periodismo de datos & 5 & Obligatoria \\
\hline 2 & Periodismo de investigación & 5 & Obligatoria \\
\hline
\end{tabular}




\begin{tabular}{clcc}
\hline Número & \multicolumn{1}{c}{ Módulo } & Créditos & Tipo \\
\hline 3 & Análisis y visualización de datos & 5 & Obligatoria \\
\hline 4 & $\begin{array}{l}\text { Laboratorio de producción audiovisual y } \\
\text { transmedia }\end{array}$ & 4 & Optativa \\
\hline 5 & $\begin{array}{l}\text { Técnicas avanzadas de análisis y } \\
\text { visualización de datos }\end{array}$ & 4 & Optativa \\
\hline 6 & Trabajo de Fin de Máster & 6 & Obligatoria \\
\hline & Fuente: Elaboración propia.
\end{tabular}

3.4. Máster universitario en periodismo digital y nuevos perfiles profesionales

Máster universitario en periodismo digital y nuevos perfiles profesionales de la URJC.

Tabla 4. Máster universitario en periodismo digital y nuevos perfiles profesionales.

\begin{tabular}{clcc}
\hline Número & \multicolumn{1}{c}{ Módulo } & Créditos & Tipo \\
\hline 6 & $\begin{array}{l}\text { Programación web para proyectos } \\
\text { periodísticos }\end{array}$ & 3 & Obligatoria \\
\hline 8 & Periodismo de datos & 6 & Obligatoria \\
\hline 9 & $\begin{array}{l}\text { Técnicas de visualización de } \\
\text { información web }\end{array}$ & 3 & Obligatoria \\
\hline 11 & Prácticas externas & 12 & Obligatoria \\
\hline 12 & Trabajo de Fin de Máster & 6 & Obligatoria \\
\hline & Fuente: Elaboración propia.
\end{tabular}

\subsection{Máster en Periodismo de Datos y Factchecking}

Máster en Periodismo de Datos y Factchecking de la Universitat Ramon Llull Blanquerna.

Table 5. Máster en Periodismo de Datos y verificación de datos.

\begin{tabular}{clcl}
\hline Módulo & \multicolumn{1}{c}{ Tema } & Créditos & Modalidad \\
\hline 1 & Introducción al tratamiento de datos & 14 & Obligatoria \\
\hline 2 & Periodismo de Datos Visualización & 10 & Obligatoria \\
\hline 3 & Fact Checking & 10 & Obligatoria \\
\hline 4 & Seminario & 5 & Obligatoria \\
\hline 5 & TFM & 9 & Obligatoria \\
\hline 6 & Prácticas en empresas & 12 & Obligatoria \\
\hline
\end{tabular}

Fuente: Elaboración propia. 


\subsection{Curso de Periodismo de Datos de El País}

Curso de Periodismo de Datos de El País y la Universidad Autónoma de Madrid (UAM).

En este caso, dado que se trata de un curso de 96 horas, se ha realizado un ajuste a créditos ECTS.

Table 6. Curso de Periodismo de Datos de El País.

\begin{tabular}{clcc}
\hline Número & \multicolumn{1}{c}{ Tema } & Créditos & Modalidad \\
\hline 1 & Introducción Periodismo Datos & 0,5 & Obligaroria \\
\hline 2 & Datos abiertos & 0,5 & Obligaroria \\
\hline 3 & Estadística & 0,5 & Obligaroria \\
\hline 4 & Hojas de cálculo & 0,5 & Obligaroria \\
\hline 5 & Bases de datos & 0,5 & Obligaroria \\
\hline 6 & Limpieza de datos & 0,5 & Obligaroria \\
\hline 7 & Web scraping & 0,5 & Obligaroria \\
\hline 8 & Visualizaciones interactivas & 0,5 & Obligaroria \\
\hline 9 & Mapas interactivos & 0,5 & Obligaroria \\
\hline 10 & Proyecto final & 0,5 & Obligaroria \\
\hline
\end{tabular}

Fuente: Elaboración propia.

\subsection{Máster propio UC3M-EFE en Periodismo de Agencia}

Máster en Periodismo de Agencia UC3M-EFE.

Table 7. Máster propio UC3M-EFE en Periodismo de Agencia.

\begin{tabular}{cccc}
\hline Número & Tema & Créditos & Modalidad \\
\hline 4 & Periodismo de datos & 6 & Obligaroria \\
\hline
\end{tabular}

\subsection{Máster de Periodismo y Visualización de Datos}

Máster de Periodismo y Visualización de Datos de la Universidad de Alcalá (UAH).

Table 8. Máster de Periodismo y Visualización de Datos.

\begin{tabular}{clcc}
\hline Número & \multicolumn{1}{c}{ Tema } & Créditos & Modalidad \\
\hline 1 & Fundamentos tecnológicos & 5 & Obligaroria \\
\hline 2 & $\begin{array}{l}\text { Introducción al Periodismo y la } \\
\text { Visualización de Datos }\end{array}$ & 5 & Obligaroria \\
\hline 3 & Organización de la información & 5 & Obligaroria \\
\hline
\end{tabular}




\begin{tabular}{clcc}
\hline Número & \multicolumn{1}{c}{ Tema } & Créditos & Modalidad \\
\hline 4 & Medios digitales & 5 & Obligaroria \\
\hline 5 & Adquisición de datos & 5 & Obligaroria \\
\hline 6 & Técnicas de tratamiento de datos & 5 & Obligaroria \\
\hline 7 & Técnicas de ciencia de datos & 5 & Obligaroria \\
\hline 8 & Sistemas interactivos en la red & 5 & Obligaroria \\
\hline 9 & Visualización interactiva & 5 & Obligaroria \\
\hline 10 & Sistemas de información geográfica & 5 & Obligaroria \\
\hline 11 & Trabajo de Fin de Máster & 10 & Obligatoria \\
\hline
\end{tabular}

Fuente: Elaboración propia.

\section{Resultados}

Los tres másteres que alcanzan los 60 créditos en temáticas de periodismo y visualización de datos son los de URJC, Blanquerna y UAH ${ }^{8}$. De estos, los dos primeros son presenciales y el último, virtual ${ }^{9}$. Otros dos, el de UOC y URJC, dedican la mitad de los créditos. Mientras, el de Nebrija dedica una cuarta parte y el de UC3M tan solo un diez por ciento. Este cálculo no puede aplicarse igual al de UAM ya que no tiene la misma duración, aunque sí que realiza un enfoque de superación de la aversión a las matemáticas. De los tres que dedican los 60 créditos cabe destacar, después de haber mirado sus programas docentes, que el de UAH aborda sin complejos los lenguajes de programación y, además, en comparación con el de UAM, emplea solo tecnologías abiertas. Ambos argumentos lo entroncan con la consideración del periodismo de datos como una parte de la ciencia de datos (Antón Bravo \& Serrano Tellería, 2020).

Table 9. Iniciativas formativas de Periodismo y Visualización de Datos.

\begin{tabular}{ccc}
\hline Universidad & Modalidad & Créditos \\
\hline URJC & Presencial & 60 \\
\hline Nebrija & Presencial & 14 \\
\hline UOC & Virtual & 29 \\
\hline URJC & Presencial & 30 \\
\hline Blanquerna & Presencial & 60 \\
\hline UAM & Presencial & 5 \\
\hline UC3M & Presencial & 6 \\
\hline UAH & Virtual & 60 \\
\hline
\end{tabular}

Fuente: Elaboración propia. 
Para emprender ese camino, el máster de UAH se plantea en los tres primeros módulos introducir el periodismo y la visualización de datos desde las tecnologías que sentaron las bases del CAR. En "Fundamentos tecnológicos" se introduce una herramienta vehicular del máster y trascendental en la relación persona-máquina como es la línea de comandos y, por extensión, las interfaces de línea de comandos. Se pretende con ello sentar las bases para aprender el lenguaje de los nuevos medios (Manovich, 2005). A continuación, se desarrolla el módulo de "Introducción al Periodismo y la Visualización de Datos" donde se hace un repaso a su historia de la materia con el objetivo de conocer los métodos y técnicas del periodismo de investigación, del periodismo de datos y de la visualización de datos. El tercer módulo, "Organización de la información", plantea el control sobre la producción propia de textos y datos. A partir del cuarto módulo y hasta el final se toma en cuenta la taxonomía OSEMN (Mason \& Wiggins, 2010) sumado al trabajo desde una perspectiva artística.

\section{Conclusiones}

Tal como ocurría con los resultados de la encuesta global de periodismo de datos (Heravi, 2018) la mayoría de las apuestas coinciden en apartados del periodismo multimedia, redes sociales o producción web y poco en los conocimientos más técnicos como la programación, estadística, ciencia de datos o aprendizaje automático. Si se abordan, lo hacen a través de aplicaciones informáticas determinadas sin una visión global de conjunto. Esta aversión a las matemáticas extendida al desconocimiento de los principios de la informática hace imposible el desarrollo y explosión esperada del periodismo y la visualización de datos como ha ocurrido en otros países.

Con la perspectiva del tiempo, el programa docente del primer máster de URJC parecía responder a las expectativas que periodistas, profesionales y medios tenían en ese momento, un escenario donde el periodismo de datos se mezclaba con la transformación digital de los medios, con la actualización de sus propuestas y con el aprendizaje basado en herramientas.

Se ha entendido el periodismo de datos, primero, como el uso de datos con un propósito periodístico para encontrar o contar historias de interés (Berret, \& Phillips, 2016). Para ello se realiza análisis de datos y su posterior narración, verificación de datos, visualización de datos o creación de aplicaciones periodísticas que ayuden a los lectores a explorar los datos por ellos mismos. En un proceso superior, el conocimiento de algoritmos, el aprendizaje automático y otras tecnologías que puedan para aprovechar la información, estructurada o no, requieren alfabetización digital. Estas cuestiones técnicas que atrajeron, en sus inicios, a los pioneros de periodismo de precisión (Meyer, 1991) y del periodismo asistido por ordenador (Cox, 2000) solo son abordadas por la UAH.

Se pretende también conectar con uno de los problemas detectados en el estudio de Davies y Cullen (2016), el de la presión de producir graduados que puedan trabajar en tareas específicas, normalmente asociadas a plataformas de producción de contenidos muy anquilosadas. En este sentido, se prefieren las apuestas por las estrategias COPE (Jacobson, 2009), APIs de entornos abiertos o de datos vinculados (Antón Bravo, 2013; Simon, 2014; McBride, 2016).

Siguiendo el planteamiento de Lev Manovich (2005), el máster de UAH entiende el trabajo con el ordenador como parte de la historia de la cultura mediática y visual de los últimos siglos y se entronca con el movimiento por el software libre y de código abierto sin servicios de terceras partes. 


\section{Agradecimientos}

Los autores agradecen a los revisores anónimos por sus comentarios y sugerencias, así como a Sara Degli-Esposti ${ }^{10}$ (CSIC) por sus oportunos consejos en las últimas etapas de este artículo.

El trabajo ha sido financiado por CSIC en el marco del proyecto Tresca ${ }^{11}$, "Trustworthy, Reliable and Engaging Scientific Communication Approaches", coordinado por Erasmus University Rotterdam y la participación de la Agencia Estatal Consejo Superior de Investigaciones Científicas (CSIC). Número de proyecto: 872855. Fecha de inicio: enero 2020. Duración: 28 meses.

El trabajo también se enmarca en el proyecto Newsnet de la convocatoria nacional de I+D+i "Restos de la sociedad", con el subproyecto cuyo título es "Noticias, redes y usuarios en el sistema mediático híbrido (Newsnet). La transformación de la industria mediática en la sociedad post industrial". Referencia: RTI2018-095775-B-C43. 3 años, 2019-2022. Ministerio de Ciencia, Innovación y Universidades.

\section{Referencias}

Anderson, C. W. (2013). Towards a sociology of computational and algorithmic journalism. New media $\mathcal{E}$ society, 15(7), 1005-1021. https://doi.org/10.1177/1461444812465137

Antón Bravo, A. (2013). El periodismo de datos y la web semántica. CIC. Cuadernos de Información y Comunicación, 18, 99-116. https://doi.org/10.5209/rev_CIYC.2013.v18.41718

Antón Bravo, A., \& Serrano Tellería, A. (2020). Data Journalism: From Social Science Techniques to Data Science Skills. Hipertext.net, (20), 41-54. https://doi.org/10.31009/hipertext.net.2020.i20.04

Bradshaw, P. (2010). How to be a data journalist. The Guardian.

Bradshaw, Paul. (2011). The Inverted Pyramid of Data Journalism. Online Journalism Blog 7.

Bradshaw, P. (2013). Data journalism heist. How to get in, get the data, and get the story out-and make sure nobody gets hurt. Leanpub, 5 .

Bradshaw, P. (2017). The online journalism handbook: Skills to survive and thrive in the digital age. Routledge.

Bradshaw, P. (2018). Data journalism teaching, fast and slow. Asia Pacific Media Educator, 28(1), 55-66. https://doi.org/10.1177/1326365X18769395

Berret, C., \& Phillips, C. (2016). Teaching data and computational journalism. Columbia School of Journalism.

Cordobés, A. I. M. (2017). El periodismo de filtraciones en España: una aproximación teórica y práctica. Los casos de Fíltrala e ICIJ en el periodismo español. In J. M. Flores (Ed.), Periodismo en nuevos formatos (pp. 2332). Fragua.

Cox, M. (2000). The development of computer-assisted reporting. Association for Education in Jornalism end Mass Comunication). Universidad de Carolina del Norte.

10https://orcid.org/0000-0003-0616-8974

11https://trescaproject.eu 
Davies, K., \& Cullen, T. (2016). Data journalism classes in Australian universities: Educators describe progress to date. Asia Pacific Media Educator, 26(2), 132-147.

Domínguez, M. Á. C. (2014). Nuevas formas informativas: el periodismo de datos y su enseñanza en el contexto universitario. Historia y comunicación social, 19(2), 43-54. https://doi.org/10.5209/rev_HICS.2014.v19.45009

Galligan, L. (2013). A systematic approach to embedding academic numeracy at university. Higher Education Research \& Development, 32(5), 734-747. https://doi.org/10.1080/07294360.2013.777037

Gray, J., Chambers, L., \& Bounegru, L. (2012). The data journalism handbook: How journalists can use data to improve the news." O'Reilly Media, Inc.

Hanusch, F., Clifford, K., Davies, K., English, P., Fulton, J., Lindgren, M., \& Zion, L. (2016). For the lifestyle and a love of creativity: Australian students' motivations for studying journalism. Media International Australia, 160(1), 101-113.

Heravi, B. R. (2018). Data journalism in 2017: A summary of results from the global data journalism survey. In G. Chowdhury, J. McLeod, V. Gillet, P. Willett (Eds.), International Conference on Information (pp. 107-113). Springer.

Investigative Journalism, International Consortium [ICIJ]. (2017). The Panama Papers Politicians, Criminals and the Rogue Industry That Hides Their Cash. https://panamapapers.icij.org/

Jacobson, D. (2009). COPE: Create Once, Publish Everywhere. https://www.programmableweb.com/news/cope-create-once-publish-everywhere/2009/10/13

Kovach, B., \& Rosenstiel, T. (2014). The Elements of Journalism: What Newspeople Should Know and the Public Should Expect. Three Rivers Press (CA).

López López, P. (2019). La enseñanza-aprendizaje del periodismo de datos en las facultades españolas: estudios y propuestas de viabiliad [Tesis Doctoral, Universidad Complutense de Madrid]. Universidad Complutense de Madrid. https://doi.org/http://dx.doi.org/10.5209/rev_CIYC.2013.v18.41718

Manovich, L. (2005). El lenguaje de los nuevos medios de comunicación. La imagen en la era digital. Paidos Comunicación.

Mason, H., \& Wiggins, C. (2010). A Taxonomy of Data Science. http://www.dataists.com/2010/09/a-taxonomyof-data-science/

Meyer, P. (1991). The new precision journalism. Indiana University Press.

Meyer, P. (2002). Precision journalism: A reporter's introduction to social science methods. Rowman \& Littlefield Publishers.

McBride, R. (2016). The ethics of data journalism [Tesis Doctoral, University of Nebraska-Lincoln]. University of Nebraska-Lincoln. https://digitalcommons.unl.edu/journalismprojects/9/

McGregor, S. (2012). On Data Literacy. https://www.youtube.com/watch?v=qGNv45xYnno 
Rogers, S. (2012). Open data journalism. The Guardian.

Simon, D. (2014). Representing Information Across Channels. In A. Resmini (Ed.), Reframing Information Architecture (pp. 131-143). Springer.

Treadwell, G., Ross, T., Lee, A., \& Lowenstein, J. K. (2016). A numbers game: Two case studies in teaching data journalism. Journalism $\mathcal{E}$ Mass Communication Educator, 71(3), 297-308. https://doi.org/10.1177/1077695816665215

Yang, F., \& Du, Y. R. (2016). Storytelling in the age of big data: Hong Kong students' readiness and attitude towards data journalism. Asia Pacific Media Educator, 26(2), 148-162. https://doi.org/10.1177/1326365X16673168

\section{@ $(0 \otimes \Theta$}

(c) Attribution-NonCommercial-NoDerivatives 4.0 International (CC BY-NC-ND 4.0) https://creativecommons.org/licenses/by-nc-nd/4.0/ 


\section{Anexo I. Enlaces de interés}

- Medialab-Prado, https://medialab-prado.es

- Primera reunión del Grupo de Trabajo de Periodismo de Datos de Medialab-Prado, https://www.medialab-prado.es/actividades/primera-reunion-del-grupo-de-trabajo-sobreperiodismo-de-datos

- JournocodersMAD, https://www.medialab-prado.es/programas/journocodersmad

- ANECA, https://www.aneca.es

- ICIJ, https://www.icij.org/

- ADALAB, https://adalab.es/

- Journocoders, https://journocoders.com/

- DataLab, https://www.medialab-prado.es/laboratorios/datalab

- Talleres de Periodismo de Datos, https://www.infotics.es/tags/tpd/

- Jornadas de Periodismo de Datos, https://www.infotics.es/tags/jpd/

- Open Knowledge Foundation Network, https://okfn.org

- Independent Reporters and Editors, https://www.ire.org/

- Iniciativa Open Source, https://opensource.org/

- Proyecto GNU, https://gnu.org

- Datawrapper, https://datawrapper.com

- Infogram, https://infogram.com

- School of Data, https://schoolofdata.org

- Data Journalism, https://datajournalism.com

- UOC, https://www.uoc.edu

- Columbia Journalism Review, https://www.cjr.org/analysis/data.php

- MPVD, https://mpvd.es/articulo/por-que-hacer-este-master/

- Tableau, https://tableau.com

- Introducción a Emacs y Orgmode, https://www.medialab-prado.es/actividades/introduccionemacs-y-orgmode

- Haskell hoy, https://www.medialab-prado.es/actividades/haskell-hoy

- Sesión veraniega del grupo de autoaprendizaje de Python, https://www.medialabprado.es/actividades/sesion-veraniega-del-grupo-de-autoaprendizaje-de-python

- Manipulación de datos y aprendizaje automático con R en el sector financiero, https://www.medialab-prado.es/actividades/manipulacion-de-datos-y-aprendizaje-automaticocon-r-en-el-sector-financiero

- Meetup de Journocoders, https://www.meetup.com/es-ES/Journocoders/ 